\title{
Polarimetic search for exoplanets with a tangential transit
}

\author{
L. V. Ksanfomality \\ Space Research Institute, Russian Academy of Sciences \\ email: ksanf@iki.rssi.ru
}

\begin{abstract}
This paper is devoted to the data obtained in measurements of the linear light polarization of few stars (that are definitely known to possess planets) and the results of the measurements processing. One may surmise with caution that the suggested method yields positive results and, if confirmed, can be of use both in searching for exoplanets in transiting or tangentially transiting orbits and in refining their masses.
\end{abstract}

Keywords. stars: planetary systems; techniques: polarimetric; methods: data analysis

\section{Introduction}

Optical polarimetry as a trial method of obtaining evidence of the presence of "hot Jupiter" planets around stars is suggested and tested experimentally by astronomical observations that have been performed. Polarimetry is convenient in its simplicity when compared with the radial velocity spectral method. Light polarization is retained in the stellar emission, irrespective of the degree of emission attenuation, unless there is a scattering medium on the line of sight.

\section{Observations and data processing}

Systematic observations of the linear light polarization of few stars (that are definitely known to possess planets) were performed in 2003 at the Steward Observatory of the University of Arizona, USA. The Kuiper telescope of Mount Bigelow Observatory $\left(110^{\circ} 44.04\right.$ $\mathrm{W}, 32^{\circ} 24.59 \mathrm{~N}$ ) has been used (a Ritchey-Chretien system, $D=1.54 \mathrm{~m}, F=20.8 \mathrm{~m}$ ). Based on the visibility conditions and the accessible duration of the observations, four short period exoplanetary systems, with an orbital period $3-4$ days, were selected: HD 46375, 51 Peg, HD 46375, Ups And and HD 209458. Among them, only HD 209458 has transits. The measurement system consisted of a Wollaston prism, filters and a cooled CCD array camera. The measurements in both components were performed simultaneously, increasing significantly the accuracy of the result. The CCD polarimeter was mounted at the prime focus of the telescope. The polarization of light from the selected stars was systematically measured within a 5 days period, in a "blue" filter (SS5). Fig. 1 shows a sequence of six polarimetric pairs of measurements of $v$ And performed with an exposure time of $220 \mathrm{~ms}$ and with intervals of $100 \mathrm{~ms}$. Since the orientation of the starlight polarization plane is not known in advance, the measurements were performed for three orientations of the Wollaston prism differed by $\alpha=45^{\circ}$. Therefore, if the light consists of a nonpolarized component $B_{n}$, and polarized component, $B_{p}$, and if the prism first have been installed at $\varphi$ angle to the light polarization plane, then the measured values in each of the two beams when the prism is turned, should vary as:

$$
b=B_{n} / 2+B_{p} \cos ^{2}(\alpha+\varphi) .
$$




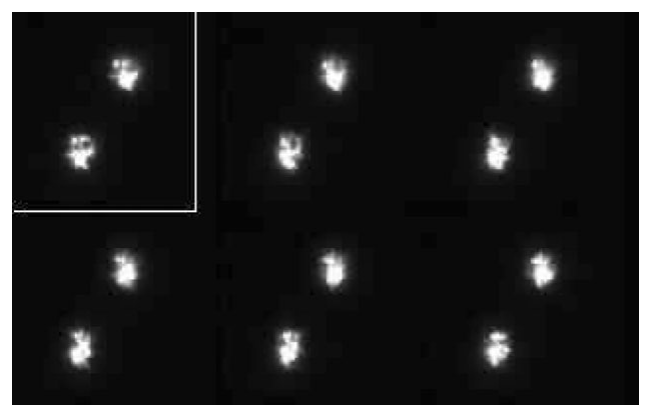

Figure 1. Example of one frame including 6 polarimetric pairs obtained during the observations of $v$ And. The position of the first pair is highlighted.

The observing time for each chosen star did not exceed $3 \mathrm{~h}$. The following data volumes have been obtained: 15.2 Mbytes in the observations of $51 \mathrm{Peg}, 12.1$ Mbytes for $v$ And, and 15.2 Mbytes for HD 209458. The data for the faint star HD 46375 was not processed later.

The ultimate goal of the data processing was the search for periodic polarization variations correlated with the known exoplanet period. More special, using the intensity ratio of orthogonally polarized waves obtained for three different groups of measurements (for different prism orientations), one could calculate the degree of polarization for the star, averaged for a given night. Once the data processing has been performed, it yields in the data like image Fig. 2. Detailed information about the processing codes is given in Talyzin (2006).

\section{Results}

Fig. 3 shows the fitting function for the observations of $v$ And and the experimental data points. Polarization and time (in days) are along the vertical and horizontal axes, respectively. It is unlikely that the scatter of points can reveal any correlation. This is also suggested by their large rms deviation from the plotted harmonic function, 0.368 . The data for the polarization variations in the star HD 209458 looks differently (Fig. 4). The rms deviation is low, 0.161 , and the data points fall nicely on the curve. Thus, the periodic polarization variations by $\pm 1.7 \%$ can be assumed to be actually attributed to the transit of the exoplanet HD 209458b and its comet-like tail across the stellar disk. The origin of the background or instrumental polarization (less than $2 \%$ ) was not investigated specially. It could be introduced by both the telescope or the camera itself.

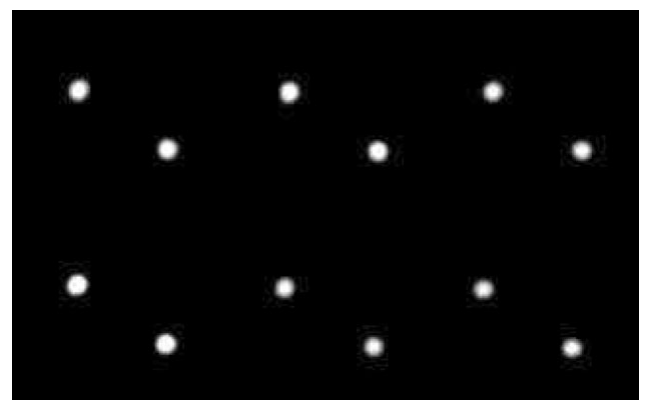

Figure 2. The image shown in Fig. 1 reconstructed from the digital array of calculated mean ratios of intensity pairs. 


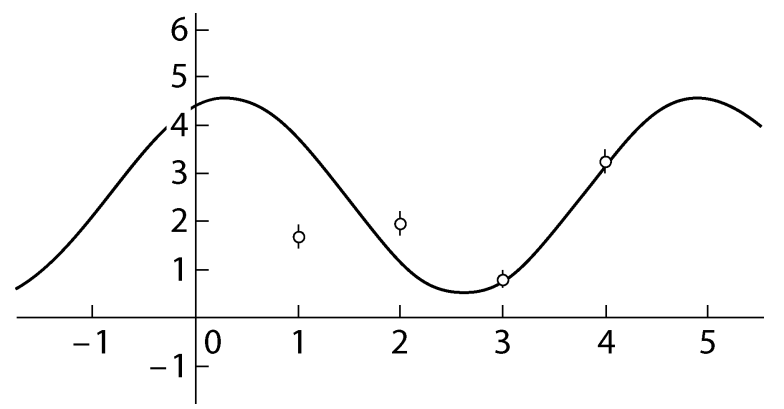

Figure 3. Search for a correlation in the data for $v$ And. Polarization (in percent) and time (in days) are along the vertical and horizontal axes, respectively. The rms deviation is 0.368 .

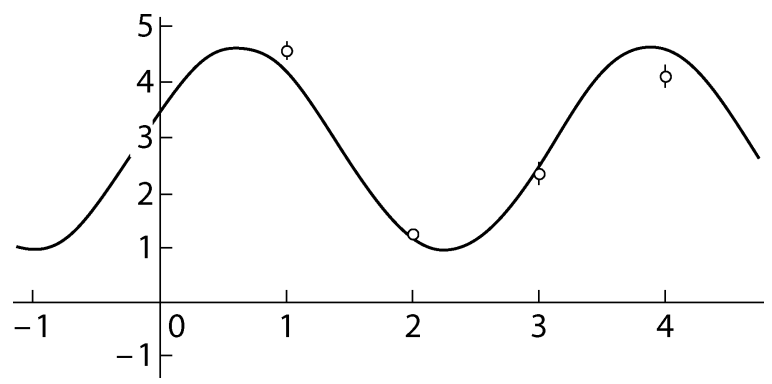

Figure 4. Polarization variations in the star HD 209458 (with exoplanet transits). The same quantities as those in Fig. 3 are along the axes. The rms deviation is 0.161 .

The most interesting is the Fig. 5 which shows the polarization variations for the star 51 Peg that is known to be the first extrasolar planet discovered (Mayor \& Queloz 1995). No transits are known for 51 Peg. Nevertheless, the rms deviation from the fitting harmonic function here is lowest, only 0.025 , while the data points show virtually almost no deviation from the curve. If this is indeed the effect of light scattering in the cometlike tail of $51 \mathrm{Peg} \mathrm{b}$, then one can assert that the angle $i$ between the orbital plane of the planet and the observer's direction is small. Hence, the exoplanet's mass $M \sin i$, known to within $\sin i$, is actually $M$, i.e., $0.46 M_{J}$ (Jupiter mass). The ultraviolet range is much more sensitive to the transits of a gaseous tail. That's why it can be suggested that ultraviolet observations will allow transits of the tail of $51 \mathrm{Peg} \mathrm{b}$ to be detected photometrically.

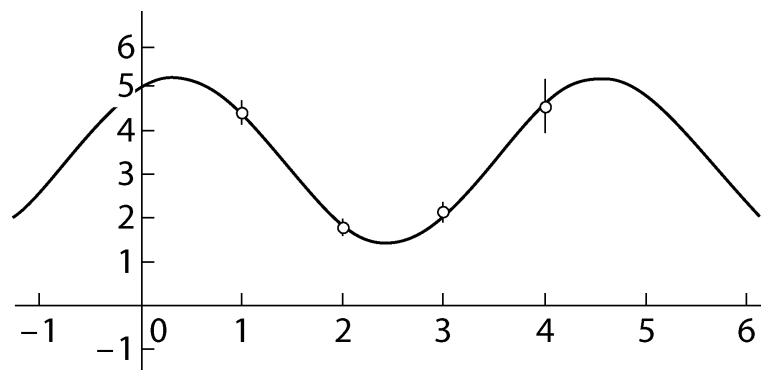

Figure 5. Polarization variations in the star $51 \mathrm{Peg}$ (the same quantities as those in Fig. 3 are along the axes). The rms deviation is 0.025 . There is no information on the transits for $51 \mathrm{Peg}$ $\mathrm{b}$ in the literature. The obtained data suggest that during the revolution of the exoplanet 51 Peg b, tangential transit of its comet-like tail across the stellar disk takes place. 
The depth of light attenuation in the UV during transits, for HD 209458 is almost an order of magnitude higher than that in the optical range.

\section{Conclusion}

The spectroscopic radial velocity method and the transit method remain the most productive for exoplanet search. Optical polarimetry is suggested as a trial method for detecting "hot Jupiter" planets around stars in transiting or tangentially transiting orbit. The method has been tested experimentally by the necessary astronomical observations and their processing (Ksanfomality 2007).

There are the following results of the first experiment (requiring confirmation by performing new astronomical observations):

- The suggested method allows to surmise with caution that it yields positive results and can be of use both in searching for exoplanets and in refining their masses.

- The possible errors cannot be ruled out completely. For example, nonidentical meteorological conditions during the observations can be the error sources.

- The high correlation between the polarization variations and the revolution period of the $51 \mathrm{Peg}$ b exoplanet suggests that the angle between the orbital plane of the planet and the observer's direction is small $(\sin i \approx 1)$ and the parameter $M \sin i$ turns into $M$. That means that the mass of first exoplanet $51 \mathrm{Peg} \mathrm{b}$ must be close to its estimated value $0.46 M_{J}$ (if the effect is actually related to a tangential transit of the exoplanet's comet-like tail).

- The above conclusions require confirmation by performing new astronomical observations to cover several complete periods of the planetary systems under study.

A possible mechanism(s) of influence of an exoplanet on the periodic variations in starlight polarization is not considered here. At least, one may suppose that light scattering in the atmosphere of an exoplanet and its comet-like tail during transit (or tangential transits) produces light polarization, same as well known effects in comet atmospheres. Apart from the light scattering in the planetary atmosphere and tail, other explanations are possible.

\section{References}

Ksanfomality, L. V. 2007, Solar System Research, 41 (4), 301.

Mayor D. \& Queloz, D. A. 1995, Nature, 378, 355.

Talyzin, A. S. 2006, Physical Peculiarities of Object HD 209458b. A New Method of Detection of Exoplanets. An Attempt to Find a Comet-like Tail of the Exoplanet in an Unusual Way. Master's Thesis (Moscow: Mosk. Fiz.-Tekh. Inst., Kafedra Kosm. Fiz.). 